\title{
El sistema de salud y sus fantasmas
}

\section{The health system and its specters}

\author{
Juan Manuel Senior • Medellín (Colombia)
}

"Lo más difícil, lo más importante. Lo más necesario, lo que a todos modos hay que intentar, es conservar la voluntad de luchar por una sociedad diferente sin caer en la interpretación paranoide de la lucha".

Estanislao Zuleta

El sistema de salud es una torre de babel, en donde convergen tan diversos intereses que dentro de sus prioridades difícilmente cabe el bienestar de las personas que acceden a él. Hacer un análisis riguroso es casi imposible, pues son demasiadas aristas; sin embargo, desde nuestra posición podemos al menos comentar las bondades y dificultades que tiene, desde la erudición que nos da la práctica del día a día, la del contacto con el paciente, la de los triunfos en sus tratamientos y la de los fracasos en las pérdidas, en las que sólo nos queda la satisfacción de haber puesto todas las herramientas terapéuticas adecuadas a su disposición y haberlo acompañado a él y a su familia.

El ejercicio de la medicina es un arte milenario, nutrido recientemente de ciencia, pero sin dejar de lado su esencia humanista, puesto que lo más importante en su concepción es el ser humano, con sus bondades y sus miserias, su familia, su entorno y sus creencias. Del médico de cabecera, aquel ser bonachón, apacible, que con tesón se enfrentaba con su estetoscopio y algunas otras herramientas rudimentarias al reto del proceso salud y enfermedad, que de igual manera atendía con dedicación a la parturienta a traer un nuevo ser en condiciones precarias, en lugares apartados; como al abuelo anciano con las vicisitudes que trae el correr de los años, que se llenaba del agradecimiento de sus pacientes y terminaba con su consultorio lleno de gallinas y otras dádivas, con el corazón pleno y el bolsillo lleno, que le permitía una vida cómoda, pasamos a inundarnos de tecnología, aquella que impone una barrera entre el médico y el paciente, a alejarnos de éstos y creer, como un sofisma real, que estamos acercando la mano de Dios en goticas.

Este "progreso", entre comillas, de la hoy llamada ciencia médica, en asocio con un sistema de salud basado en el aseguramiento, terminó por cosificar al paciente, lo convirtió en un número, en una historia, en un caso interesante y por último, desafortunadamente, en un cliente externo. ¡ Cliente! Podrían los economistas, si es que fueron ellos, haber podido inventar un vocablo más despersonalizador y humillante que éste, no, hicieron su mejor esfuerzo y lo alcanzaron. Lograron que la salud dejara de ser un derecho fundamental, que como tal es inherente a nuestra condición de ser, al solo hecho de haber nacido, puesto que muchas veces es negado antes de hacerlo, y se convirtiera en un producto que se rige por la ley de la oferta y la demanda. Esto dio pie para que entrometieran sus narices, personajes que poco saben de salud, pero sí mucho de lo que es negocio.

\section{Sistema de salud}

La Ley 100 ha estado en el banquillo desde su promulgación, aunque cuenta con adeptos que la defienden, son muchos los enemigos y detractores que destacan sus falencias. Hay que resaltar dentro de sus logros, el aumento de la cobertura en salud de una franja de la población menos favorecida, aquellas personas que inundaban las mal llamadas salas de caridad de nuestros antiguos hospitales; sin embargo, esto no ha sido suficiente y en muchos casos
Dr. Juan Manuel Senior: Presidente Asociación Colombiana de Medicina Interna. Medellín (Colombia).

Correspondencia. Dr. Juan Manuel Senior. Medellín (Colombia).

E-mail: mmbt@une.net.co

Recibido: 26/II/2012 Aceptado: 26/III/2012 
sólo se convirtió en un proceso de carnetizacion sin un real acceso a los servicios de salud y en otros casos a atención de dudosa calidad, puesto que para el sistema son importantes los números pero no el resultado, permitiendo la proliferación de centros de atencion montados en garajes o casas viejas, lejos de una infraestructura adecuada, sin ningún control.

El sistema basado en el aseguramiento convierte un derecho fundamental en el privilegio de algunos pocos, que acceden al sistema porque tienen capacidad de pago y quienes carecen de recursos lo hacen por la magnanimidad de los primeros.

El Estado descuidó por completo la vigilancia de los indicadores de salud importantes que permitían establecer las condiciones de la población, y es así como numerosas enfermedades objeto de este seguimiento fueron descuidadas y su reaparición motivo de controversia. Hecho a todas luces predecible, dado que el sistema afecta en forma adversa este tipo de acciones, privilegiando la ganancia económica por encima del bienestar poblacional.

Un sistema que adjudica a todos los actores por igual, sin tener en cuenta calidad ni resultados, minimiza la importancia de éstos como objetivo final del trabajo y de la inversión, básicamente porque no logra estimular la obtención de resultados tales como la disminución de la prevalencia de enfermedades, la disminución de la mortalidad en grupos específicos o al menos mantener las cifras actuales. Porque las acciones en promoción y prevención se limitan a consultas periódicas por personal de enfermería o médicos generales y a la realización de algunos exámenes sin un real impacto en las tasas de prevalencia e incidencia.

De igual forma se permitió la creación de muchas empresas (EPS, EPSS) sin el debido respaldo y vigilancia, que se convirtieron en fortines políticos en el mejor de los casos o en nido de corruptos en el peor. Muchas de estas empresas pobremente vigiladas, hicieron y deshicieron con el dinero del sistema, favorecieron familiares en forma directa o indirecta, hecho bastante reprochable y deplorable en cualquier empresa, contubernio de amigos y foco de corrupción, desangrando las pobres finanzas y llevando a la tardía intervención del Estado o a la quiebra de éstas. El negocio entonces no solamente está en la función de intermediario, sino en la fácil desviación de los recursos para beneficio propio. El negocio permite que si para usted no es rentable puede abdicar y devolverle al Estado sus afiliados, como sucede actualmente con una franja de pacientes subsidiados o sisbenizados.

Un aspecto importante por discutir es la creencia de que la promoción y prevención es un proceso barato o al menos que obtiene réditos económicos; sin embargo, esta concepción está muy alejada de la realidad. La conformación de un verdadero programa de promoción y prevención en salud implica el despliegue de campañas masivas, que nada tienen de económicas, generan una mejor cultura en salud, propenden por un diagnóstico más temprano y por lo tanto de mejores resultados del acto médico, lo que implica un gasto económico enorme, un número de pacientes mayor para atender, necesidad de tecnología de punta en el diagnóstico y más y mejores insumos.

Obviamente es la estrategia que mayor impacto tiene en indicadores como mortalidad, calidad de vida y días laborales perdidos, por mencionar algunos. El sistema en forma maquiavélica permite que sea más barato la pérdida de una vida humana que la atencion de un enfermo, razón por la cual Colombia podría ser uno de los pocos países en donde sobran órganos para donación y faltan receptores. Es inexplicable por qué, tal vez con la excepción del riñón, se pierden órganos en el proceso de donación multiorgánica. La tasa de trasplante renal se vio impulsada por la entrada del nuevo sistema de salud, puesto que es más costoso mantener en diálisis a un paciente por un año, tres veces por semana, que el trasplante y la inmunosupresión por el mismo periodo, diferente del paciente con falla cardiaca que muchas veces fallece sin siquiera conocer la posibilidad de que un trasplante hubiese cambiado su evolución y sobrevida.

Desde este punto de vista, el desarrollo de guías de atención integral basadas en la evidencia, podría convertirse en un aliado formidable para cambiar el panorama en este aspecto. Si el esfuerzo que realiza el ministerio con distintas entidades para dar a la luz pública un número apreciable de estos instrumentos logra implementarse, la situación podría cambiar. Si éstas sólo se quedan en documentos fríos, archivados en anaqueles o publicados en revistas científicas, sin que exista un verdadero programa de implementación que genere cambios en las conductas médicas y mediante indicadores específicos se detecte el efecto real de las recomendaciones en la población objeto de la guía, el esfuerzo será absolutamente inútil y por ende costoso.

Otro aspecto relevante, es la calidad de los medicamentos disponibles en el mercado. No es una apología a los medicamentos de marca y una diatriba contra los genéricos, es sólo la búsqueda de medicamentos de calidad que permitan replicar los resultados obtenidos en estudios de investigación multicéntricos. Disponemos en el mercado de un sinnúmero de medicamentos de dudosa procedencia, cuyo efecto dista mucho del medicamento original y muchas veces es menor que la de cualquier placebo.

Creo que todos coincidimos en este concepto y conocemos pacientes con reacciones como la que acabo de describir. Es famoso el efecto de un anticoagulante genérico que disminuye los niveles de INR a valores normales, a pesar de aumentar el número de pastillas administradas, puesto que no es un real incremento de la dosis, y de la consabida sobreanticoagulación cuando se cambia este esperpento farmacológico por el original en la misma cantidad. Otro medicamento genérico inmunosupresor que disminuye los valores medidos del metabolito activo en pacientes trasplantados y por ende induce mayores tasas de rechazo y pone en grave riesgo la sobrevida del órgano y del paciente. Podríamos seguir con una lista interminable de estas especies, que desafortunadamente no son anecdóticas. 
Es importante para cierto tipo de medicamentos conocer no sólo datos de su bioequivalencia sino de su equivalencia clínica, que en último término es el factor crucial. Ciertos medicamentos tienen procesos complejos de manufacturación que son difíciles de replicar en condiciones diferentes, puesto que no sólo es la estructura química lo que importa, esto ha llevado en el mundo a la comercialización de insumos de pobre calidad, proveniente de lugares de dudosa reputación. La idea no es tampoco exigir replicar el estudio clínico que demostró las bondades del nuevo fármaco, pero sí la respuesta en una población específica y la vigilancia de sus efectos clínicos y eventos adversos.

No es menos cierto que algunos actores del sistema han abusado de los precios de medicamentos con indicaciones específicas y sin alternativas en el mercado. Explotando por muchos años al sistema y reduciendo costos en forma dramática de un momento a otro, por motivos de regulación o por mayor competencia o quien sabe por qué otras razones.

\section{Ejercicio médico}

La autonomía médica es el pilar del ejercicio médico honesto y ético. Dejar que factores externos influyan en la toma de decisiones en momentos puntuales o durante todo el proceso es absolutamente catastrófico y adicionalmente sugerir que las conductas tomadas pueden ser punibles, un desacierto mayúsculo, el cual sólo cabe en cerebros con problemas en su sinapsis. Desafortunadamente en el día a día, nos vemos enfrentados a este tipo de situaciones, que muy difícilmente pueden ser denunciadas o expuestas en forma clara, dado que sus protagonistas las enmascaran en procesos administrativos, todos absolutamente legales.

Una de las estrategias es condicionar la remuneración que recibe el médico a su tasa de remisiones a especialistas, de formulación de medicamentos de alto costo o al número de paraclínicos ordenados, vieja táctica importada de países australes, aunque dudo del significado de austral, que prosperó durante algunos años y se mimetizó en forma de bonificación. Otra no menos importante, es la determinación del tiempo de consulta de medicina general o de especialista en 15 a 20 minutos, adicionándole trabas administrativas, como por ejemplo la formulación de sólo tres medicamentos por fórmula, lo que obliga a llenar el encabezado con interminables datos, cuando usted se excede y en lo cual ocupa el $70 \%$ del tiempo, lo que genera múltiples quejas de los usuarios, otro gran vocablo, porque sólo acceden a la opinión distante de un funcionario de la EPS y a medicamentos como el acetaminofén, al ácido acetil salicílico o al diclofenaco, sea cual fuere su motivo de consulta, por poner sólo un ejemplo. Detrás de éstas hay otra lista de acciones administrativas, no menos insultantes, como llamados de atención por no remitir pacientes a un nivel inferior, por no aumentar la tasa de consultas nuevas, por reasignar citas, puesto que lo importante es que se atienda al paciente no importa cómo ni con qué calidad.
Esa obtusa idea encarece la atencion, puesto que un profesional es incapaz de hacer una adecuada disquisición clínica con los elementos recogidos de la entrevista y el examen físico del paciente y se refugia en una serie de exámenes, muchas veces innecesarios o no indicados, llevando a diagnósticos errados y por lo tanto pobre respuesta terapéutica.

Estas y otras situaciones han deteriorado la calidad de vida del profesional en medicina y sus ingresos, obligándolo a asumir conductas cuyo riesgo no ha sido estimado. Asumir la responsabilidad de la atención de cuatro pacientes por hora, con medicamentos de dudoso efecto, con tecnología inadecuada, asumir la responsabilidad de la realización de procedimientos quirúrgicos o de intervención en las mismas condiciones por remuneraciones insultantes, que lo llevan a doblar su horario de trabajo o a realizar turnos de dobles jornadas o a trabajar en horas nocturnas en una institución y en horario diurno en otra, como si hubiese dormido en su propia cama toda la noche, sin pensar que cualquier error podría costarle no sólo la pérdida de una vida, sino todo el esfuerzo de su propia vida, su patrimonio, y convertirlo en un paria de la sociedad.

No hemos sido ajenos a la idea de la salud como negocio, puesto que muchos colegas decidieron, como forma de protegerse contra este inhumano mercado, volverse dueños, invertir, comprar equipos, manejar las finanzas. Hecho desde todo punto de vista respetable, pero que sin embargo entraña riesgos importantes en la práctica profesional. ¿Qué protegemos en situaciones coyunturales, el paciente o nuestra inversión? ¿Cuál es la conducta adecuada cuando se llega la hora de pagar el leasing o el préstamo bancario y el aparato, la máquina o el dispositivo no ha generado los recursos esperados? ¿Cuál es la opción correcta cuando se ofrecen ganancias adicionales por la utilización de uno u otro insumo? Estas inquietudes plantean situaciones complicadas de resolver y analizar en tan solo unas líneas; sin embargo, debemos reconocer que la mayoría de nuestros colegas han sabido afrontarlas en forma ética y responsable con sus pacientes y el sistema.

Macartizar las relaciones entre la industria y el gremio médico, las asociaciones científicas y los propios colegas ha sido otra estrategia utilizada, por un ministro de ingrata recordación, para explicar el caos administrativo y el desangre económico. Esa soterrada insinuación alcanzó a tomar fuerza y fuimos casi expuestos al escarnio público, con la propuesta de reforma de la salud presentada para aprobación en el anterior gobierno, que afortunadamente las asociaciones científicas, unidas y representadas por la Asociacion de Sociedades Científicas y las diferentes partes interesadas logramos hundir. No podemos desconocer que existen relaciones oscuras, poco éticas entre el gremio y la industria, unas fomentadas por estas mismas, otras por nuestros propios colegas, pero es un total desacierto generalizar tal despropósito y mancillar nuestro buen nombre.

Una manera socorrida de retrasar o bloquear el ejercicio médico es contratar un enjambre de colegas, la mayoría de 
las veces de pobre rendimiento académico en su pregrado, para auditar como par, las decisiones de sus colegas y menos comprensible, la de sus profesores. Siempre tendrán dudas que resolver, interrogantes sobre las indicaciones y obviamente análisis económicos sobre la terapia escogida. En esta relación perversa con los auditores a través del sistema, se pierde el respeto y el colegaje, porque se tiene un patrón diferente, con objetivos diferentes; dentro de esta relación caben acciones inconcebibles, como trasladar un paciente de una institución a otra, porque los costos en aquélla son menores, exponiendo al paciente a un riesgo innecesario, ante la mirada inerme de las instituciones de salud. Es lógico que un enfermo se traslade de una institución a otra o de una ciudad a otra cuando no se le pueda ofrecer la terapia adecuada, en cuyo caso el riesgo del transporte está plenamente justificado, pero hacerlo sólo porque la contratación es diferente, raya con la ética y la mala práctica. Es de rescatar que estas estrategias no son utilizadas por todas las empresas prestadoras de salud, aunque sí por una gran proporción, ni por todos los colegas.

El juego de la libre oferta y demanda ha producido la liquidación de varias históricas instituciones de salud en el país, llevadas al borde del abismo por la inequidad del sistema. En forma inocente muchas IPS hacen contratos leoninos, algunas veces llamados por capitación, lo que les da flujo de caja y por lo tanto un respiro económico temporal; sin embargo, en escaso tiempo se ven inundados y sobrepasados por los pacientes más enfermos y por lo tanto los que más recursos consumen, convirtiéndose en un dolor de cabeza. En otros casos su institución es absorbida por pacientes de una sola empresa, hecho que es facturado en una próxima negociación, pues en el mercado es bastante peligroso depender de un solo actor. En la época de renegociar le dejan su sitio de trabajo desierto, sin pacientes, en forma sigilosa, como una amenaza subliminal, logrando disminuir las cifras en varios ceros al cerrar la nueva contratación.

\section{Formación médica}

Las universidades distan de ser refugios de académicos, pues su capacidad de atraer nuevos prospectos es bastante limitada. La posibilidad de atraer cerebros fugados es irrisoria, la diferencia en los salarios ofrecidos es abismal, más evidente en personas con posgrados o doctorados. El sistema de estímulos es adverso y los comités de asignación de puntajes se convierten en "carteles" para desestimular la producción académica, única posibilidad de mejorar la remuneración. Es tan evidente el desconocimiento de la producción académica que artículos o capítulos de libro publicados, son calificados en diferente forma por las diversas universidades, algunos de ellos productos de asociaciones científicas como ACMI ${ }^{\circledR}$ o la Sociedad Colombiana de Cardiología son calificados como impresos universitarios, sin que medie reclamo alguno, generando diferencias en los salarios de profesores con similar tiempo de vinculación y producción de acuerdo con la región del país. También es cierto que en algunas de ellas se excedieron en la asignación de puntajes, poniendo en peligro la estabilidad económica de la institución.

A pesar de la evidente falta de especialistas, en algunas áreas, la ineficiencia estatal produce que el problema esté lejos de resolverse y las iniciativas institucionales sean desestimuladas. El tiempo de aprobación de un posgrado en promedio es alrededor de tres años para universidades de reconocido prestigio y la obtención del registro calificado de programas ya aprobados otro tanto. Por lo anterior, la tan mencionada autonomía universitaria es una falacia. Los programas viajan de oficina en oficina, de ministerio en ministerio y de funcionario en funcionario, hasta que son devueltos con pocas explicaciones, y cuando se pregunta, la respuesta es aún más desconcertante: "No se preocupen que generalmente los documentos se devuelven varias veces".

Esta situación es el causante del pobre ofrecimiento de programas de posgrado en diversas áreas, puesto que a pesar de que contamos con un número no despreciable de maestrías y doctorados en ciencias básicas, existe una absoluta carencia de éstos en áreas clínicas. De igual manera existe déficit de especialistas en algunas áreas; sin embargo, este hecho es magnificado por las EPS, para escudar su ineficiencia administrativa y los bajos salarios ofrecidos a estos profesionales. Todavía más desconcertante la propuesta ministerial hace algunos años de encargar a médicos generales de la atencion de especialidades básicas como la medicina interna, entre otras, desconociendo por completo la esencia de nuestra especialidad.

La pregunta siguiente es cómo se logra la aprobación de programas completos de pregrado de instituciones sin historia en el área medicocientífica, sin ninguna experiencia en el tema, que mediante engaños atraen a incautos bachilleres que ven en ellas una posibilidad de estudio, y por lo tanto se ven en la penosa obligación de recurrir a promociones, para quienes remitan o atraigan estudiantes a sus claustros, bastante vergonzoso ofrecer premios, aunque absolutamente legal, y cuyos lemas parecen amenazas escalofriantes como que usted tarde o temprano estudiará aquí, o programas claramente ligados a la actividad de empresas prestadoras de salud, cuyo objetivo dista de ser éste, nuevamente legal, jugando el rol de juez y parte. Sin duda, este tipo de programas ha deteriorado la calidad de la medicina en Colombia.

\section{Desinformación de la población}

Un aspecto en que consetudinariamente fallamos las asociaciones científicas, es en la proyección a la comunidad. Nuestra labor de educación a la comunidad es ínfima y la hemos dejado en manos de personas sin conocimiento y experiencia, en el mejor de los casos. Estamos inundados de programas televisivos cuyo objetivo es desinformar y promocionar cápsulas, ungüentos y otras especies cuyos efectos son milagrosos. Son innumerables los centros de medicina alternativa cuyo rédito económico no está en la evaluación o la consulta del paciente sino en los productos 
que venden, por tal razón pasamos de los resultados milagrosos del jugo de una fruta asiática, al té verde de Ho Chi Min hasta las cápsulas rejuvenecedoras, promocionada por una mujer histriónica, de curvas sugestivas y evidente grupo etáreo. O la evaluación con aparatos de nombres ininteligibles que al analizar su iris o el hígado o la lengua u otro órgano rector, expulsa un resultado fantástico seguido por una receta mágica.

¿Por qué no existe una regulación clara para este tipo de productos, cuyo respaldo científico es nulo y sus efectos secundarios desconocidos? ¿Por qué el Estado permite que la población esté expuesta a este tipo de riesgos? Entiéndase que no es un ataque frontal a la medicina alternativa, es un llamado a la reflexión y a la reglamentación para cumplir con lineamientos científicos y legales exigidos para todos los productos que tienen que ver con la salud. Es menos entendible que una institución universitaria participe en este tipo de actividades en forma similar. De igual forma, fármacos con indicaciones específicas, como el omega 3 , por poner un ejemplo, son promocionados para indicaciones no probadas, generando falsas expectativas y echando mano del socorrido efecto placebo.

\section{Educación médica continua}

Es loable el esfuerzo que realizamos las asociaciones científicas para mantener actualizados a nuestros colegas en los avances recientes en diferentes áreas. Sin embargo, este tipo de esfuerzos nunca tiene el apoyo estatal suficiente o por parte de las empresas prestadoras de salud, que en forma errónea lo ven como una amenaza, puesto que si sus médicos se actualizan, aprenderán indicaciones de nuevas drogas, más potentes, que ofrecen mejores resultados, pero siempre son más costosas.

De esta manera, siempre bloquean los permisos para asistir a seminarios, talleres y congresos, obligando a los colegas a invertir parte de su tiempo libre en esas actividades, incluso en días posteriores a la realización de turnos nocturnos. Muchas veces se ofrecen a estas empresas inscripciones gratuitas que son desaprovechadas.

La educación médica continua la enmascaran en algunas conferencias dictadas por médicos de la misma empresa, cuya actualización también es deficiente, y obviamente son registradas y exhibidas como prueba fehaciente de que sus médicos sí cumplen con los requisitos exigidos para el cargo y están debidamente actualizados. Como consecuencia, se genera otro problema aún más inquietante, algunos colegas son actualizados por la visita de los representantes de la industria farmacéutica.

El proceso de acreditación en educación médica que hemos acometido algunas asociaciones científicas nos permitirá formular un programa de recertificación que permita mantener los niveles de idoneidad y erigirnos como defensores a ultranza de la excelencia académica, especialmente en medicina interna y sus subespecialidades.

Dentro de todo este aparente caos hay acciones y actores sumamente rescatables que pueden permitir mejorar el sistema y frenar su progresivo deterioro. Por lo tanto estas opiniones, que no son más que reflexiones producto de las tribulaciones del ejercicio médico actual, es una invitación a reformular el sistema de salud, con el concurso de todos; Estado, asociaciones, médicos, pacientes, universidades e industria con el único objetivo de lograr el bien común. 